\title{
Editorial
}

\section{Development of Computed Tomography Algorithms}

\author{
Hengyong Yu, ${ }^{1}$ Patrick J. La Rivière, ${ }^{2}$ and Xiangyang Tang ${ }^{3}$ \\ ${ }^{1}$ Department of Radiology, University of Iowa, Iowa City, IA 52242, USA \\ ${ }^{2}$ Department of Radiology, University of Chicago, Chicago, IL 60637, USA \\ ${ }^{3}$ Applied Science Laboratory, GE Healthcare, Waukesha, WI 53188, USA
}

Received 31 May 2006; Accepted 31 May 2006

Copyright (c) 2006 Hengyong Yu et al. This is an open access article distributed under the Creative Commons Attribution License, which permits unrestricted use, distribution, and reproduction in any medium, provided the original work is properly cited.

Over the past several years, computed tomography (CT) methods have advanced significantly, yielding novel analytic and iterative solutions applicable to medical CT and micro-CT. The resulting algorithms promise to improve spatial, contrast, or temporal resolution as well as to suppress artifacts and reduce radiation dose. Significant attention has been devoted to optimizing computational performance and to balancing conflicting requirements. Both theoretically oriented and application-specific issues are also being addressed. As a snapshot of the dynamically changing field of $\mathrm{CT}$, this special issue includes 10 high-quality original papers.

Because spiral cone-beam CT can be used for rapid volumetric imaging with high longitudinal resolution, the development of exact and efficient algorithms for image reconstruction from spiral cone-beam projection data has been a subject of active research in recent years. Katsevich's filtered backprojection (FBP) formula represents a significant breakthrough in this field [1]. In this special issue, Yang et al. propose a parallel implementation of Katsevich's FBP formula [2] by the one-beam cover method, in which the backprojection procedure is independently driven by cone-beam projections. Based on Katsevich's work, generalized backprojection filtration (BPF) and FBP algorithms are developed to reconstruct images from data collected along more flexible scanning trajectories [3]. Using these recently developed algorithms, Yu et al. propose a local region reconstruction scheme [4]. The principal idea is to deliver a normal radiation dose to a local region of interest (ROI) that may contain a lesion while applying a very low radiation dose to the structures outside the ROI. Both the FBP and BPF algorithms can produce excellent results with a minimal increment to the dose needed for purely local CT.

Despite important advancements in the development of exact cone-beam reconstruction, approximate algorithms remain practically and theoretically valuable. Feldkamp et al. heuristically adapted the fan-beam FBP algorithm for approximate cone-beam reconstruction in the case of a circular scanning locus [5]. This formulation, called the FDK algorithm, is more desirable in many cases than exact conebeam reconstruction approaches in terms of several aspects of image quality and computational implementation. Since then, many efforts have been made to extend the FDK algorithm to other scanning configurations, leading to a series of FDK-like algorithms. In this special issue, Yan et al. propose an approximate FDK-like reconstruction algorithm for tilted-gantry CT imaging [6]. The method improves the image reconstruction by filtering the projection data along a direction that is determined by CT parameters and the tiltedgantry angle. Based on the idea that there is less redundancy for the projection data away from the central scanning plane, Yang and Ning develop a heuristic cone-beam geometric dependent weighting scheme [7], which leads to a new FDKlike half-scan algorithm. For correcting cone-beam artifacts in off-centered geometry, Valton et al. compare and evaluate four different reconstruction methods [8], which are the Alpha-FDK algorithm, a shift-invariant FBP method derived from the T-FDK, an FBP method based on the Grangeat formula, and an iterative algebraic method. Tang et al. extend the $3 \mathrm{D}$ weighted helical CB-FBP algorithm to handle helical pitches that are lower than $1: 1$ [9]. For helical over-scan, the extended 3D weighted helical CB-FBP algorithm can significantly improve noise characteristics or dose efficiency compared to the original algorithm, while other advantages of the original algorithm, such as reconstruction accuracy and computational efficiency, can be maintained.

In addition to the exact and approximate CBCT algorithms, iterative algorithms are important technologies in medical X-ray CT. It is well known that a major weakness of the noniterative algorithms, either exact or approximate, is that projection data are implicitly assumed to be 
noise-free. However, noise is an inherent aspect of projection data, especially for low-dose scans. Iterative algorithms are well suited to deal with image artifacts caused by photon noise or other physical effects. Qi compares maximum a posteriori (MAP) reconstructions with Gaussian and nonGaussian priors [10]. After evaluating three representative priors: the Gaussian prior, the Huber prior, and the GemanMcClure prior, Qi concludes that the Gaussian prior is as effective as the more complex non-Gaussian priors for lesion detection and quantification tasks. Rather than performing full-blown iterative reconstruction involving projecting and reprojecting the image, La Riviere et al. explore iterative approaches to sinogram restoration followed by analytic reconstruction. Here they compare the use of quadraticand median-based roughness penalties [11], and they find that the two approaches produce similar resolution-variance tradeoffs to each other, which suggests that the particular choice of penalty may be less important than the decision to use a penalty at all. Israel-Jost et al. propose build frequencyadapted (FA) algorithms based on a condition of incomplete backprojection [12], leading to an FA-simultaneous algebraic reconstruction technique (FA-SART) algorithm. The results obtained with the FA-SART algorithm on a highly detailed phantom demonstrate a very fast convergence compared to the original SART algorithm.

The recent advances in X-ray technology provide highcontrast and spatiotemporal resolution, which offer new potential for evaluation of cardiac kinetics with 4D dynamic sequences. In this special issue, Garreau et al. propose a new method for cardiac motion extraction in multislice CT based on a $4 \mathrm{D}$ hierarchical surface-volume matching process [13]. Their aim is to detect the left heart cavities along the acquired sequence and estimate their 3D surface velocity fields. A Markov random field model is defined to find, according to topological descriptors, the best correspondences between a 3D mesh describing the left endocardium at one time-point and the 3D acquired volume at the following time-point. The global optimization of the correspondences is realized with a multiresolution process.

In closing this introduction to the special issue, we would like to express our appreciation to all the authors and reviewers for the tremendous efforts that have made the timely completion of our assignment successful and pleasant. Hope this special issue would attract a major attention of the peers and inspire more creative research ideas in the CT field.

Hengyong Yu

Patrick J. La Riviere Xiangyang Tang

\section{REFERENCES}

[1] A. Katsevich, "An improved exact filtered backprojection algorithm for spiral computed tomography," Advances in Applied Mathematics, vol. 32, no. 4, pp. 681-697, 2004.

[2] J. Yang, X. Guo, Q. Kong, T. Zhou, and M. Jiang, "Parallel implementation of theKatsevich's FBP algorithm," International
Journal of Biomedical Imaging, vol. 2006, Article ID 17463, 8 pages, 2006.

[3] S. Zhao, H. Yu, and G. Wang, "A unified framework for exact cone-beam reconstruction formulas," Medical Physics, vol. 32, no. 6, pp. 1712-1721, 2005.

[4] H. Yu, Y. Ye, S. Zhao, and G. Wang, "Local ROI reconstruction via generalized FBP and BPF algorithms along more flexible curves," International Journal of Biomedical Imaging, vol. 2006, Article ID 14989, 7 pages, 2006.

[5] L. A. Feldkamp, L. C. Davis, and J. W. Kress, "Practical conebeam algorithm," Journal of the Optical Society of America A, vol. 1, no. 6, pp. 612-619, 1984.

[6] M. Yan, C. Zhang, and H. Liang, "An approximate cone beam reconstruction algorithm for gantry-tilted CT using tangential filtering," International Journal of Biomedical Imaging, vol. 2006, Article ID 29370, 8 pages, 2006.

[7] D. Yang and R. Ning, "FDK half-scan with a heuristic weighting scheme on a flat panel detector-based cone beam CT (FDKHSCW)," to appear in International Journal of Biomedical Imaging.

[8] S. Valton, F. Peyrin, and D. Sappey-Marinier, "Analysis of cone beam artifacts in off-centered circular CT for four reconstruction methods," to appear in International Journal of Biomedical Imaging.

[9] S. M. McOlash, R. A. Nilsen, J. Hsieh, and X. Tang, "Extending three-dimensional weighted cone beam filtered backprojection (CB-FBP) algorithm for image reconstruction in volumetric CT at low helical pitches," to appear in International Journal of Biomedical Imaging.

[10] J. Qi, "Comparison of lesion detection and quantification in MAP reconstruction with Gaussian and non-Gaussian priors," International Journal of Biomedical Imaging, vol. 2006, Article ID 87567, 10 pages, 2006.

[11] P. J. La Riviere, J. Bian, and P. A. Vargas, "Comparison of quadratic- and median-based roughness penalties for penalized-likelihood sinogram restoration in computed tomography," International Journal of Biomedical Imaging, vol. 2006, Article ID 41380, 7 pages, 2006.

[12] V. Israel-Jost, P. Choquet, and A. Constantinesco, "A prospective study on algorithms adapted to the spatial frequency in tomography," International Journal of Biomedical Imaging, vol. 2006, Article ID 34043, 6 pages, 2006.

[13] M. Garreau, A. Simon, D. Boulmier, J.-L. Coatrieux, and H. Le Breton, "Assessment of left ventricular function in cardiac MSCT imaging by a 4D hierarchical surface-volume matching process," International Journal of Biomedical Imaging, vol. 2006, Article ID 37607, 10 pages, 2006.

Hengyong Yu received his B.S. degree in information science and technology and Ph.D. degree in information and communication engineering from Xi'an Jiaotong University, Xi'an, Shaanxi, China, in July 1998 and June 2003, respectively. He was an Instructor and Associate Professor with the College of Communication Engineering, Hangzhou Dianzi University, Hangzhou, Zhejiang, China, from July 2003 to Septem-

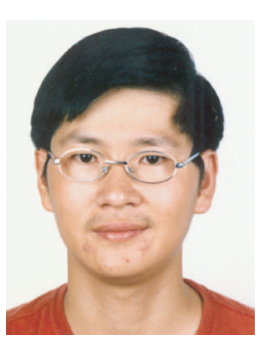
ber 2004. Currently, he is an Associate Research Scientist, Associate Director of the CT/Micro-CT lab, Department of Radiology, University of Iowa, Iowa City, USA. His interests include computed tomography and medical image processing. He has authored or coauthored more than 30 peer-reviewed papers. He is a Senior Member 
of the IEEE. He was honored for an outstanding doctorial dissertation by Xi' an Jiaotong University, and received the First Prize for a Best Natural Science Paper from the Association of Sci. \& Tech. of Zhejiang Province.

Patrick J. La Riviere received the A.B. degree in physics from Harvard University in 1994 and the Ph.D. degree from the Graduate Programs in Medical Physics in the Department of Radiology at the University of Chicago in 2000. In between, he studied the history and philosophy of physics while on the Lionel de Jersey-Harvard Scholarship to Cambridge University. He is currently an Assistant Professor in the Department of

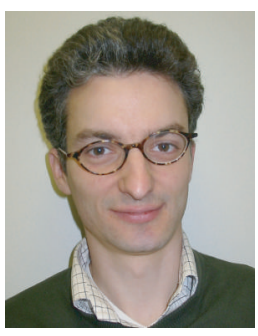
Radiology at the University of Chicago, where his research interests include tomographic reconstruction in computed tomography, Xray fluorescence computed tomography, and optoacoustic tomography.

Xiangyang Tang is currently with the Applied Science Laboratory of GE Healthcare Technologies as a Senior Scientist. With a focus on the development of image reconstruction algorithms for cone beam (CB) volumetric computed tomography (VCT) in diagnostic imaging, he has been working in the field of CB-VCT for almost 10 years, covering system analysis and design, development of algorithms for system cali-

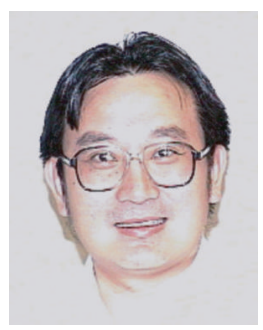
bration, artifacts suppression, digital image/video processing, and CT cardiac imaging applications. In the recent 5 years, as an active Researcher in the field of medical imaging, he published more than 50 papers or book chapters in scientific journals and conference proceedings, and filed more than 10 US patent applications. Meanwhile, he also served as Guest Editor or Reviewer for a number of prestigious scientific journals in the field of medical imaging. 

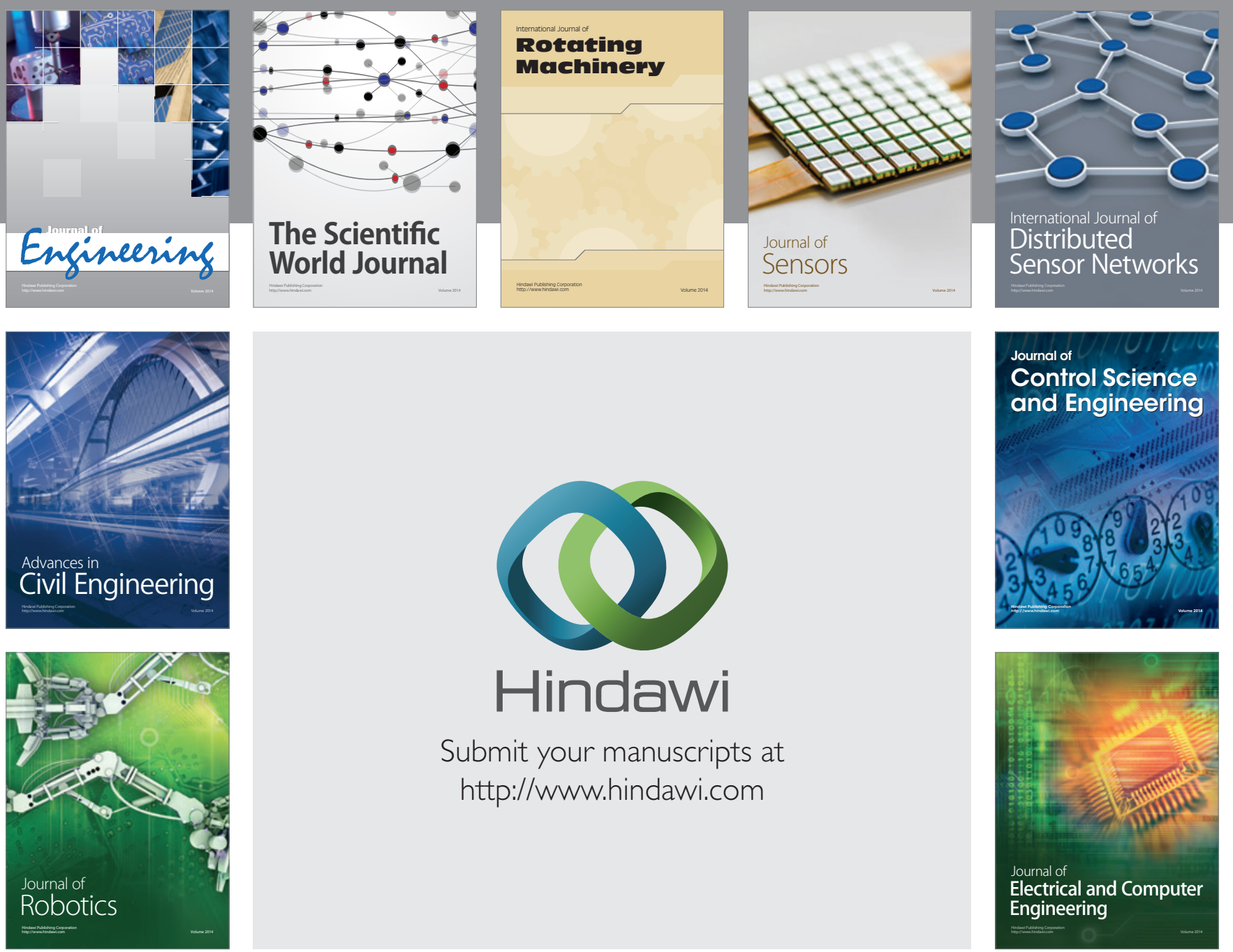

Submit your manuscripts at

http://www.hindawi.com
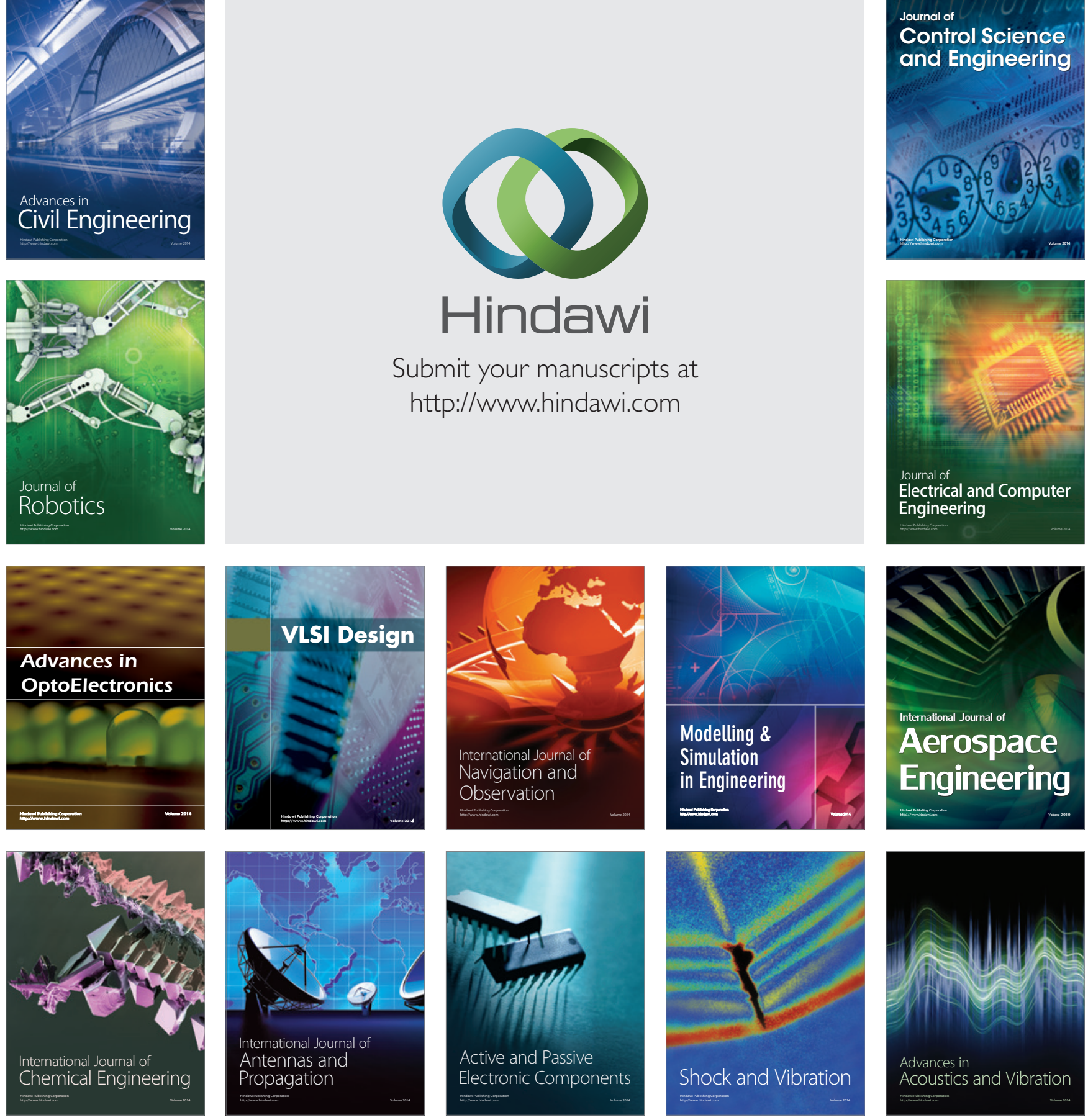